\title{
Qualidade do Sistema de Informações sobre Nascidos Vivos (Sinasc): análise crítica da literatura
}

\author{
Quality of the Information System on Live Births /SINASC: \\ a critical analysis of published studies
}

${ }^{1}$ Programa de PósGraduação em Saúde Pública, Pró-Reitoria de Pós-Graduação e Pesquisa, Universidade Estadual da Paraíba. Av. das Baraúnas 351/Campus Universitário, Bairro Bodocongó. 58109-753 Campina Grande Paraíba. dixisfigueroa@gmail.com
Abstract The scope of this study was to carry out a review of scientific literature about the quality of data from the Information System on Live Births (SINASC). Studies in the Medline, Lilacs and SciELO databases were then reviewed. The articles were characterized according to journal of publication, location and period of the study, evaluation parameters, methodology and main results. A total of thirteen articles were reviewed. Eight studies analyzed coverage, four assessed completeness and seven evaluated reliability. Coverage higher than $90 \%$ was presented in the majority of the studies, indicating its viability for the calculation of indicators. However, the underreporting of births in SINASC prevailed with variation between $75.8 \%$ and $99.5 \%$. The mother's educational level, number of prior childbirths and frequency of prenatal visits were the variables that led to greater inconsistency. For its part, the parity variable was the one that led to greater incompleteness. In conclusion, the development of studies to find new ways of assessing SINASC is highlighted as a privileged strategy for the enhancement of the quality of the system.

Key words Vital statistics, Information systems, Live birth, Brazil
Resumo Objetivo: Realizar uma revisão da literatura sobre os estudos de avaliação da qualidade dos dados do Sistema de Informações sobre Nascidos Vivos (Sinasc). Métodos: As bases de dados Medline, Lilacs e SciELO foram pesquisadas. Os artigos foram caracterizados segundo revista escolhida para publicação, local e período de estudo, parâmetros de avaliação, metodologia e principais resultados. Resultados: Um total de 13 artigos foi revisado. A avaliação da cobertura foi tema de análise em oito estudos, a completitude em quatro e a confiabilidade em sete. A maioria deles apresentou resultados de cobertura superior a 90\%, indicando sua viabilidade para o cálculo de indicadores. Entretanto, prevaleceu o sub-registro dos nascimentos no Sinasc com variação entre 75,8\% e 99,5\%. As variáveis instrução materna, paridade e número de consultas de pré-natal foram as que mostraram maior inconsistência. Por sua vez, a variável paridade foi a que mostrou maior incompletitude. Conclusões: O desenvolvimento de estudos para encontrar novas formas de avaliar o Sinasc é destacado como estratégia privilegiada para a melhoria da qualidade do sistema.

Palavras-chave Estatísticas vitais, Sistemas de informação, Nascidos vivos, Brasil 


\section{Introdução}

No Brasil, os registros de nascimentos, até o início da década de 90, estiveram baseados exclusivamente no Sistema de Registro Civil. Estes compreendiam apenas os nascimentos que eram informados em cartório, com níveis variáveis de sub-registro, de acordo com as regiões do país. Entretanto, o reconhecimento da importância das informações sobre os nascimentos vivos para as estatísticas de saúde, epidemiologia e demografia, levou o Ministério da Saúde do Brasil a implantar, em 1990, o Sistema de Informações sobre Nascidos Vivos (Sinasc). O sistema, de âmbito nacional e sob a responsabilidade das Secretarias Municipais e Estaduais de Saúde, foi implantado com o objetivo principal de conhecer o perfil epidemiológico dos nascimentos vivos, segundo variáveis como peso ao nascer, duração da gestação, tipo de parto, idade da mãe e número de partos ${ }^{1}$.

O Sinasc não foi introduzido de forma homogênea em todo o país. Sua implantação ocorreu de forma gradual entre as Unidades da Federação, de forma que seus dados foram divulgados apenas a partir de $1994^{2}$. Com a implantação do Sinasc, dispõe-se, assim, pela primeira vez, em nível municipal, estadual e nacional, não apenas de informações sobre nascimentos ocorridos no ano, mas também de informações sobre as mães, a gravidez, o parto e o recém-nascido. Dessa forma, é possível obter um panorama epidemiológico mais detalhado e espacialmente desagregado das condições de saúde materno-infantili ${ }^{2,3}$.

O Sinasc baseia-se nos dados contidos na Declaração de Nascido Vivo (DNV), documento oficial e padronizado para todo o país. A DNV é de emissão obrigatória nos hospitais e outras instituições de saúde nos quais são realizados partos. Os Cartórios do Registro Civil também apresentam tal obrigatoriedade relacionada com os partos ocorridos no domicílio e cuja informação chega a esse tipo de instituição ${ }^{1,3}$. O primeiro modelo de DNV era formado por oito blocos de informação. Em janeiro de 1996, um novo modelo circulava no país, com algumas mudanças em relação ao anterior. Alterações sucessivas modificaram a forma de algumas perguntas, suprimiram algumas questões (exemplo: nome do pai) ou introduziram novas variáveis (exemplo: raça/ cor, presença de anomalia congênita) $)^{3}$.

O modelo em vigor da DNV está disponível na Internet ${ }^{4}$. O fluxo de encaminhamento das declarações, as normas quanto ao seu preenchimento e o processamento das informações, são aspectos contemplados no Manual de Procedimentos $^{5}$ e no Manual de Instruções para o Preenchimento da $\mathrm{DNV}^{4}$. As informações sobre os nascidos vivos são disponibilizadas para a população por meio da página da Internet do Datasus. Os dados devem estar disponíveis em um prazo de, no máximo, dois anos ${ }^{2}$.

Os estudos científicos realizados a partir dos sistemas de informação em saúde possuem desvantagens ligadas à utilização de dados secundários (como o viés de informação, por exemplo). Entretanto, possuem como vantagem a realização, a baixo custo, de estudos de base populacional e de abrangência nacional ${ }^{6}$. Assim, o Sinasc constitui-se atualmente em uma ferramenta essencial para o conhecimento do perfil epidemiológico, o planejamento e a avaliação das ações de saúde materno-infantil. É um instrumento extremamente valioso para caracterizar a situação do parto e do nascimento, as mães e seus bebês. A qual, permite acompanhar o perfil deste segmento da população e traçar políticas de saúde adequadas, voltadas para grupos específicos, de acordo com perfil de risco ${ }^{1,2}$. Por outro lado, é um dos componentes principais para alcançar a equidade, a qualidade e a humanização dos serviços de saúde, assim como para fortalecer o controle social no âmbito do Sistema Único de Saúde (SUS) ${ }^{7}$.

No entanto, a consolidação do sistema como fonte de dados de grande relevância para a saúde pública exige esforços relacionados à melhoria das informações. Isto redunda na necessidade de avaliar as informações do Sinasc, seja do ponto de vista quantitativo (cobertura do sistema, completitude do preenchimento), quanto qualitativo (confiabilidade/fidedignidade das informações). A qualidade das informações é fundamental para o uso do sistema como fonte de informação relevante para pesquisa e avaliação em saúde. Assim, as análises etiológicas e os indicadores derivados das informações contidas no Sinasc devem refletir a realidade do perfil de uma determinada população. Em função da implantação descentralizada do Sinasc, é preciso, também, que o desenvolvimento de avaliações do sistema compreenda os níveis estaduais e municipais ${ }^{3,7}$.

Considerando esses pressupostos, o objetivo do presente estudo foi realizar uma análise e discussão crítica da literatura sobre qualidade dos dados do Sinasc. Busca-se, dessa forma, permitir uma melhor compreensão do que pode ser pensado como qualidade no Sinasc e que estudos poderiam ser feitos para melhorar o seu conhecimento. 


\section{Métodos}

Para a identificação dos artigos, realizou-se, em março de 2010, uma busca ativa de informações nas bases de dados Medline, Lilacs e SciELO. A pesquisa bibliográfica considerou o tema central deste trabalho: estudos de avaliação da qualidade dos dados do Sinasc. Com a finalidade de delimitar o objeto de estudo e o campo de investigação para a realidade que se pretende apreender, optou-se por selecionar apenas produções na forma de artigos publicados em periódicos. O ano de 1990 foi considerado como o período de início para tal levantamento, pois foi neste ano que ocorreu a implantação do Sinasc.

Os termos utilizados para a busca de artigos foram: "estatísticas vitais" e "sistemas de informação”, utilizando a interseção dos conjuntos. A busca foi realizada nos idiomas português e inglês. No caso da busca no Medline o descritor Brazil também foi usado.

Para o cômputo do total de estudos identificados foi verificada a duplicação entre as bases de dados, sendo cada artigo contabilizado somente uma vez. A partir dos estudos identificados, considerando a leitura dos títulos e resumos, foram selecionados aqueles artigos originais (delineados) abordando a avaliação do Sinasc. Todos os artigos selecionados foram avaliados considerando a leitura e a análise criteriosa do texto completo. Após esta ação, foram excluídos artigos claramente não relacionados com o tema (sem menção ao Sinasc), artigos abordando a avaliação da qualidade de outros sistemas de informação, artigos de revisão ou tese e artigos sem a utilização de indicadores de qualidade (cobertura, completitude, fidedignidade).

Com o objetivo de localizar artigos que não tivessem sido encontrados na busca inicial, a lista de referências dos estudos incluídos foi utilizada para a identificação de outros artigos. Estes, também foram avaliados respeitando os critérios de seleção.

Os artigos selecionados foram caracterizados segundo revista escolhida para publicação, local e período de realização da pesquisa, parâmetro(s) de avaliação (cobertura, completitude, fidedignidade), metodologia e principais resultados.

\section{Resultados}

Inicialmente foram identificados 170 artigos. Com a leitura dos títulos e resumos, em um pri- meiro momento, e dos artigos na sua íntegra, posteriormente, foram incluídos um total de nove artigos que adequadamente preenchiam todos os critérios de seleção. A consulta à lista de referências identificou outros quatro artigos que preenchiam adequadamente esses critérios. $\mathrm{O}$ fluxo do número de estudos incluídos encontra-se mostrado na Tabela 1.

Foram analisados $13 \operatorname{artigos}^{1,2,7-17}$. A Tabela 2 mostra sua distribuição quanto aos parâmetros de caracterização adotados. Todos os estudos foram publicados em periódicos da área de Saúde Coletiva, tendo os Cadernos de Saúde Pública $(\mathrm{n}=7)$ o maior volume de publicações. Os outros periódicos com artigos publicados foram a Revista de Saúde Pública $(\mathrm{n}=4)$ e a Revista Epidemiologia e Serviços de Saúde $(n=2)$. Observase, assim, uma concentração quanto à escolha do periódico para a divulgação de estudos avaliativos da qualidade dos dados do Sinasc.

A maioria dos estudos foi desenvolvida em âmbito municipal $(\mathrm{n}=9)^{1,8,9,11-13,15-17}$. Entretanto, uma pesquisa foi realizada, considerando os dados de todo o território nacional, abrangendo as cinco regiões do país ${ }^{7}$. O estado federativo onde houve maior frequência de estudos foi São Paulo $(\mathrm{n}=3)^{12,15,17}$. O Nordeste foi a região que concentrou maior quantidade de estudos $(\mathrm{n}=5)^{2,8,11,13,14}$.

Tabela 1. Etapas da seleção, exclusão e inclusão de artigos na revisão sobre estudos de avaliação da qualidade dos dados do Sistema de Informações sobre Nascidos Vivos (SINASC).

\begin{tabular}{lc}
\hline \multicolumn{1}{c}{ Etapas/Critérios de exclusão e inclusão } & $\begin{array}{c}\text { Número } \\
\text { de artigos }\end{array}$ \\
\hline Artigos identificados & 140 \\
Medline & 32 \\
Lilacs & 07 \\
SciELO & 09 \\
Estudos em duplicata & 170 \\
Total de estudos identificados & 151 \\
Estudos excluídos & 01 \\
- claramente não relacionados com o tema & 01 \\
- avaliação da qualidade de outros sistemas de informação & 04 \\
- artigos de revisão & 04 \\
- tese & 161 \\
- não oferecem indicadores de qualidade & \\
Total de estudos excluídos & 09 \\
Estudos incluídos (busca nas bases de dados) & 04 \\
Lista de referências & 13 \\
Total de estudos incluídos & \\
\hline
\end{tabular}


Tabela 2. Características dos artigos sobre avaliação da qualidade dos dados do Sistema de Informações sobre Nascidos Vivos (SINASC).

\begin{tabular}{|c|c|c|c|c|c|}
\hline Autor, ano & $\begin{array}{l}\text { Revista de } \\
\text { publicação }\end{array}$ & $\begin{array}{l}\text { Local, período } \\
\text { de estudo }\end{array}$ & $\begin{array}{l}\text { Parâmetro(s) } \\
\text { de avaliação }\end{array}$ & Metodologia & Principais resultados \\
\hline $\begin{array}{l}\text { Costa e } \\
\text { Frias }^{2}\end{array}$ & $\begin{array}{l}\text { Cad Saude } \\
\text { Pública }\end{array}$ & $\begin{array}{l}\text { Pernambuco, } \\
1996-2005\end{array}$ & Completitude & $\begin{array}{l}\text { Cálculo do percentual } \\
\text { de incompletitude } \\
\text { (preenchimento em } \\
\text { branco + ignorado) e } \\
\text { construção de séries } \\
\text { temporais para cada } \\
\text { variável }\end{array}$ & $\begin{array}{l}\text { - Maioria dos municípios (> } \\
95 \% \text { ) com preenchimento } \\
\text { excelente das variáveis } \\
\text { - Aumento na \% de municípios } \\
\text { com preenchimento excelente } \\
\text { com destaque para as variáveis } \\
\text { raça/cor da criança, instrução } \\
\text { materna, estado civil e número } \\
\text { de consultas de pré-natal } \\
\text { - Variáveis com maior } \\
\text { incompletitude: índice de Apgar } \\
\text { no } 1^{\circ} \text { e no } 5^{\circ} \text { minuto, paridade }\end{array}$ \\
\hline Silva et al. ${ }^{8}$ & $\begin{array}{l}\text { Cad Saude } \\
\text { Publica }\end{array}$ & $\begin{array}{l}\text { Maracanaú } \\
(\mathrm{CE}) \text {, janeiro } \\
2000 \text {-dezembro } \\
2002\end{array}$ & Cobertura & $\begin{array}{l}\text { Pareamento do } \\
\text { SINASC e o SIM }\end{array}$ & $\begin{array}{l}\text { - Subregistro dos nascimentos no } \\
\text { SINASC (falhas na emissão da } \\
\text { Declaração de Nascido Vivo) }\end{array}$ \\
\hline $\begin{array}{l}\text { Guerra } \\
\text { et al. }{ }^{9}\end{array}$ & $\begin{array}{l}\text { Cad Saude } \\
\text { Publica }\end{array}$ & $\begin{array}{l}\text { Rio de Janeiro } \\
(\mathrm{RJ}), 2004\end{array}$ & Confiabilidade & $\begin{array}{l}\text { Comparação da } \\
\text { Declaração de Nascido } \\
\text { Vivo com os } \\
\text { prontuários hospitalares } \\
\text { das crianças e das mães }\end{array}$ & $\begin{array}{l}\text { - Variáveis com menor } \\
\text { consistência: instrução materna, } \\
\text { presença de anomalias } \\
\text { congênitas } \\
\text { - Obs.: Valores de consistência } \\
\text { bastante variados conforme o } \\
\text { tipo de defeito congênito, sendo } \\
\text { em alguns casos bem expressivos }\end{array}$ \\
\hline $\begin{array}{l}\text { Drumond } \\
\text { et al. } .^{10}\end{array}$ & $\begin{array}{l}\text { Rev Saude } \\
\text { Publica }\end{array}$ & $\begin{array}{l}\text { Minas Gerais, } \\
2001\end{array}$ & Cobertura & $\begin{array}{l}\text { Comparação do } \\
\text { número de nascidos } \\
\text { vivos do SINASC e o } \\
\text { SIH Relacionamento } \\
\text { probabilístico de dados } \\
\text { individuais do SINASC } \\
\text { e o SIH }\end{array}$ & $\begin{array}{l}\text { Comparação de eventos } \\
\text { - 98,8\% (Minas Gerais) } \\
\text { - 95,6\% (Municípios de pequeno } \\
\text { porte) } \\
\text { - 97,6\% (Municípios de porte } \\
\text { intermediário 1) } \\
\text { - 97,5\% (Municípios de porte } \\
\text { intermediário 2) } \\
\text { - 100,0\% (Municípios de grande } \\
\text { porte) } \\
\text { Probabilístico } \\
\text { - 91,6\% (Minas Gerais) } \\
\text { - 85,7\% (Municípios de pequeno } \\
\text { porte) } \\
\text { - 88,3\% (Municípios de porte } \\
\text { intermediário 1) } \\
\text { - 89,4\% (Municípios de porte } \\
\text { intermediário 2) } \\
\text { - 93,8\% (Municípios de grande } \\
\text { porte) } \\
\text { - Melhor identificação de } \\
\text { subnotificação no SINASC } \\
\text { através do relacionamento } \\
\text { probabilístico }\end{array}$ \\
\hline
\end{tabular}


Tabela 2. continuação

\begin{tabular}{|c|c|c|c|c|c|}
\hline Autor, ano & $\begin{array}{l}\text { Revista de } \\
\text { publicação }\end{array}$ & $\begin{array}{l}\text { Local, período } \\
\text { de estudo }\end{array}$ & $\begin{array}{c}\text { Parâmetro(s) } \\
\text { de avaliação }\end{array}$ & Metodologia & Principais resultados \\
\hline $\begin{array}{l}\text { Romero e } \\
\text { Cunha }^{7}\end{array}$ & $\begin{array}{l}\text { Cad Saude } \\
\text { Publica }\end{array}$ & Brasil, 2002 & Confiabilidade & $\begin{array}{l}\text { - Cálculo do percentual } \\
\text { de incompletitude } \\
\text { (preenchimento em } \\
\text { branco + ignorado) - } \\
\text { Pareamento do } \\
\text { SINASC e Censo } \\
\text { Demográfico } 2000\end{array}$ & $\begin{array}{l}\text { - Maioria das variáveis com boa } \\
\text { completitude e consistência } \\
\text { - Variáveis com maior } \\
\text { incompletitude: paridade, raça/ } \\
\text { cor da criança, ocupação materna } \\
\text { - Variáveis com menor } \\
\text { consistência: paridade, raça/cor } \\
\text { da criança, ocupação materna }\end{array}$ \\
\hline Frias et al. ${ }^{11}$ & $\begin{array}{l}\text { Epidemiol } \\
\text { Serv Saúde }\end{array}$ & $\begin{array}{l}\text { Bom Conselho, } \\
\text { Brejo da Madre } \\
\text { de Deus (PE), } \\
\text { dezembro } \\
\text { 1999-maio } \\
2000\end{array}$ & Cobertura & $\begin{array}{l}\text { Pareamento do } \\
\text { SINASC e SINASC + } \\
\text { busca ativa (registro das } \\
\text { unidades de saúde, } \\
\text { agente comunitário de } \\
\text { saúde, parteira) }\end{array}$ & $\begin{array}{l}\text { - 89,1\% (Bom Conselho) } \\
\text { - } 97,1 \% \text { (Brejo) }\end{array}$ \\
\hline $\begin{array}{l}\text { Almeida } \\
\text { et al. }{ }^{12}\end{array}$ & $\begin{array}{l}\text { Cad Saude } \\
\text { Publica }\end{array}$ & $\begin{array}{l}\text { São Paulo, } \\
\text { região sul (SP), } \\
\text { agosto 2000- } \\
\text { fevereiro } 2001\end{array}$ & Confiabilidade & $\begin{array}{l}\text { - Cálculo do percentual } \\
\text { de incompletitude } \\
\text { (preenchimento em } \\
\text { branco + ignorado) } \\
\text { - Pareamento do tipo } \\
\text { caso-controle do } \\
\text { SINASC e pesquisa } \\
\text { sobre mortalidade } \\
\text { perinatal }\end{array}$ & $\begin{array}{l}\text { - Variáveis com maior } \\
\text { incompletitude: paridade, } \\
\text { presença de anomalias } \\
\text { congênitas } \\
\text { - Variáveis com menor } \\
\text { consistência: instrução materna, } \\
\text { situação conjugal } \\
\text { - Obs.: Não foi determinada a } \\
\text { confiabilidade para as variáveis } \\
\text { com alto \% de incompletitude } \\
\text { (paridade e presença de } \\
\text { anomalias congênitas) }\end{array}$ \\
\hline $\begin{array}{l}\text { Theme } \\
\text { et al. }{ }^{1}\end{array}$ & $\begin{array}{l}\text { Cad Saude } \\
\text { Publica }\end{array}$ & $\begin{array}{l}\text { Rio de Janeiro } \\
(\mathrm{RJ}) \text {, } \\
1999-2001\end{array}$ & $\begin{array}{l}\text { Cobertura } \\
\text { Confiabilidade }\end{array}$ & $\begin{array}{l}\text { Pareamento do } \\
\text { SINASC e pesquisa } \\
\text { sobre morbi- } \\
\text { mortalidade e da } \\
\text { atenção peri e neonatal }\end{array}$ & $\begin{array}{l}\text { - 96,5\% de cobertura } \\
\text { - Variáveis com menor } \\
\text { consistência: situação conjugal, } \\
\text { instrução materna, número de } \\
\text { consultas de pré-natal }\end{array}$ \\
\hline Silva et al. ${ }^{13}$ & $\begin{array}{l}\text { Rev Saude } \\
\text { Publica }\end{array}$ & $\begin{array}{l}\text { São Luis (MA), } \\
\text { 1997-1998 }\end{array}$ & $\begin{array}{l}\text { Cobertura } \\
\text { Confiabilidade } \\
\text { Completitude }\end{array}$ & $\begin{array}{l}\text { - Pareamento do } \\
\text { SINASC e pesquisa } \\
\text { perinatal } \\
\text { - Cálculo do percentual } \\
\text { de incompletitude } \\
\text { (preenchimento em } \\
\text { branco + ignorado) }\end{array}$ & $\begin{array}{l}\text { - 75,8\% de cobertura } \\
\text { - Variáveis com menor } \\
\text { consistência: idade gestacional, } \\
\text { instrução materna, número de } \\
\text { consultas de pré-natal, paridade } \\
\text { - Variáveis com maior } \\
\text { incompletitude: idade } \\
\text { gestacional, instrução materna, } \\
\text { número de consultas de pré- } \\
\text { natal, paridade }\end{array}$ \\
\hline $\begin{array}{l}\text { Mello Jorge } \\
\text { e Gotlieb }\end{array}$ & $\begin{array}{l}\text { Inf Epidemiol } \\
\text { SUS }^{*}\end{array}$ & $\begin{array}{l}\text { Sergipe, Ceará e } \\
\text { Tocantins, } \\
1998\end{array}$ & Cobertura & $\begin{array}{l}\text { Pareamento do } \\
\text { SINASC e o SIAB }\end{array}$ & $\begin{array}{l}\text { - } 150 \% \text { (Ceará) } \\
\text { - 210\% (Sergipe) } \\
\text { - 270\% (Tocantins) } \\
\text { - Subregistro dos nascimentos no } \\
\text { SINASC em alguns municípios }\end{array}$ \\
\hline
\end{tabular}




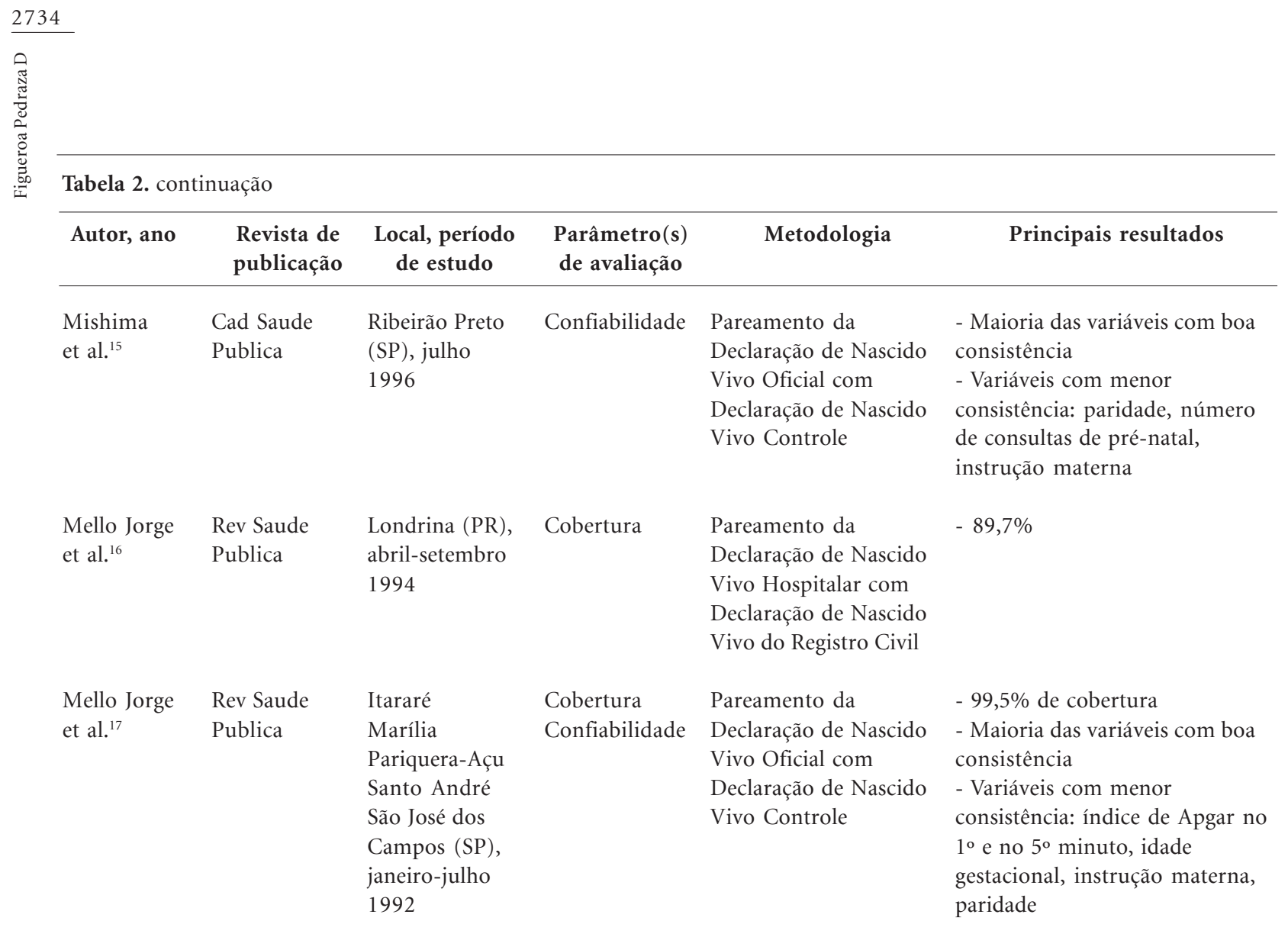

SIM: Sistema de Informações sobre Mortalidade. SIH: Sistema de Informação Hospitalar. SIAB: Sistema de Informação da Atenção Básica.

*A Revista Informe Epidemiológico do SUS cessa em 2002 e passa a se chamar Epidemiologia e Serviços de Saúde.

De acordo com os parâmetros de avaliação, constatou-se que oito artigos ${ }^{1,8,10,11,13,14,16,17}$ $(61,5 \%)$ estudaram a cobertura, quatro ${ }^{2,7,12,13}$ $(30,8 \%)$ a completitude e sete $\mathrm{e}^{1,7,9,12,13,15,17}(53,8 \%)$ a confiabilidade dos dados. Na mensuração do grau de cobertura, usaram-se como possíveis bases de dados mais confiáveis o registro civil ${ }^{16}$, outros sistemas de informação (SIM, SIH, SIAB $)^{8,10,14}$, dados primários ${ }^{1,13}$, dados contro$1 \mathrm{e}^{17}$ e a busca ativa complementar aos dados do próprio sistema ${ }^{11}$. Na mensuração da completitude, todos os estudos ${ }^{2,7,12,13}$ consideraram a proporção de dados não em branco e ignorados. $\mathrm{Na}$ mensuração da confiabilidade, usou-se para pareamento as informações fornecidas por outras fontes secundárias ${ }^{9}$, dados primários ${ }^{1,7,12,13}$ e dados controle $e^{15,17}$

A avaliação da cobertura indicou, majoritariamente, sub-registro dos nascimentos no Sinasc, mostrando variação entre $75,8 \%{ }^{13}$ e $99,5 \%{ }^{17}$ nos casos em que o indicador foi calculado. A maior cobertura do Sinasc foi encontrada no estudo que avaliou o parâmetro considerando os dados do $\mathrm{SIAB}^{14}$ e nos municípios de grande porte de Minas Gerais considerando os dados do $\mathrm{SIH}^{10}$.
Com relação à análise de confiabilidade e completitude, observa-se que foram estudadas 14 variáveis, sendo instrução materna ${ }^{1,9,12,13,15,17}$, paridade (referente ao número total de filhos anteriores: vivos e mortos) $)^{1,7,13,15}$ e número de consultas de pré-natal ${ }^{1,13,15}$ as que mostraram maior inconsistência. A variável paridade ${ }^{2,7,12,13}$ foi a que mostrou maior incompletitude. As variáveis índice Apgar ${ }^{2,17}$, raça/cor da criança ${ }^{2,7}$, ocupação materna ${ }^{7}$ e presença de anomalias congênitas $^{9,12}$ foram destacadas como inconsistentes e incompletas no mínimo uma vez.

No estudo de abrangência nacional, realizado por Romero e Cunha ${ }^{7}$ referente ao ano 2002, os autores agruparam o país por região geográfica e por unidades da federação, analisando a qualidade do Sinasc quanto à completitude e confiabilidade. Adicionalmente, foi avaliada a clareza metodológica das instruções de coleta e preenchimento, assim como a correlação da completitude com indicadores de pobreza, desigualdade econômica e recursos humanos em saúde. Os autores apontam que o "Manual de instruções para o preenchimento da declaração de nascido vivo" é confuso quanto aos códigos para infor- 
mação ignorada e quando não se aplica. Além disso, indicam que as variáveis raça/cor do recém-nascido e ocupação materna não são claras por falta de especificação sobre quem deve ser o informante, no caso da primeira, e por problemas de definição, no caso da segunda. Paridade e as duas variáveis anteriores foram as que apresentaram maior incompletitude e menor consistência. Bahia, Sergipe, Distrito Federal, Maranhão, Ceará e Goiás foram as unidades federativas com maiores problemas no quesito completitude. Menor consistência foi encontrada para as variáveis total de filhos nascidos vivos (no Distrito Federal e no Rio de Janeiro), raça/cor (no Norte e no Nordeste) e ocupação materna (no Nordeste). Por intermédio da análise estatística, observou-se relação significativa entre a completitude dos dados e os indicadores de pobreza e desigualdade.

Mishima et al. ${ }^{15}$ e Guerra et al. ${ }^{9}$ foram os únicos autores que analisaram o fluxo de preenchimento da DNV. Eles verificaram que o fluxo é heterogêneo entre os hospitais, envolvendo o médico, enfermeiro, auxiliar de enfermagem, escrituraria de berçário e funcionários administrativos. Também destacam que o preenchimento foi realizado por diversos profissionais dependendo da variável.

\section{Discussão}

Com a disponibilização dos dados do Sinasc no sítio do Datasus a partir de 1994 e o reconhecimento da importância do sistema para a avaliação e gestão em saúde, era esperado que houvesse um crescimento nos períodos mais recentes das publicações voltadas para a avaliação da qualidade (cobertura, confiabilidade, completitude) das informações fornecidas através do Sinasc.

Os resultados obtidos expressam concentração da produção científica segundo o veículo de publicação e o local de estudo. É de ressaltar que nenhum dos estudos analisou a região Norte. Os dados anteriores corroboram os resultados obtidos por pesquisas em outros temas de saú$\mathrm{de}^{6}$. Este fato pode estar relacionado ao número de grupos de pesquisa e instituições de ensino superior nas diferentes regiões do país.

A melhoria na qualidade das informações fornecidas através do Sinasc implica na necessidade de aumentar as atividades de supervisão e controle de qualidade da coleta e processamento de dados, redefinindo as variáveis com problemas metodológicos e incentivando os profissio- nais encarregados pelo completo e correto preenchimento. Nesse contexto, e considerando as variáveis que se mostraram com maiores problemas de confiabilidade e completitude, alguns pontos importantes devem ser considerados na busca de soluções que visem melhorar a qualidade dos dados do Sinasc $\mathrm{S}^{2,3,7,13}$ :

1. Pouca clareza metodológica do manual de preenchimento da DNV;

2. O manual de instruções para o preenchimento da DNV é confuso quanto aos códigos para informação ignorada e quando não se aplica a pergunta, a exemplo do que acontece com a variável paridade;

3. Heterogeneidade de profissionais responsáveis pelo preenchimento da DNV, muitos dos quais não estão qualificados para a função;

4. Maior interesse em determinadas variáveis em detrimento de outras, a exemplo do que pode acontecer com a variável instrução materna;

5. Mau preenchimento dos prontuários do recém-nascido e da puérpera, documentos mais utilizados como fonte de dados para o preenchimento da DNV;

6. Erros de grafia e na transcrição das informações, a exemplo do que pode acontecer com a variável paridade materna.

A análise desses fatores sugere a necessidade de implantação de atividades de capacitação das equipes técnicas dos setores envolvidos na coleta, processamento e divulgação das informações, seja na esfera federal, estadual ou municipal, para a melhoria da qualidade dos dados do Sinasc. Nesse sentido, os gestores municipais devem ser motivados, sensibilizados e orientados quanto ao uso dos dados de nascidos vivos na construção de indicadores apropriados, capazes de fornecer subsídios para análise adequada da situação de saúde no nível local. Como resultado da descentralização de serviços de saúde, as análises devem ser realizadas considerando os diferentes níveis de desagregação, possibilitando o estabelecimento de metas a serem adotadas pelos níveis locais. Assim, outro ponto relevante é que os gestores dos sistemas de informação devem aproveitar as experiências exitosas de várias localidades e promover, sempre que necessário, investigações visando à melhoria da qualidade dos dados ${ }^{3,7}$.

Adicionalmente, na análise da qualidade das informações, o grau de desenvolvimento do município ou unidade federativa deve ser ponderado como possível fator relacionado às diferenças observadas entre os estudos ${ }^{7}$. Dessa forma, a demanda pela qualificação dos dados do Sinasc, com ampliação da cobertura e maior qualidade no 
preenchimento das variáveis, implica na necessidade de investimentos em infraestrutura.

Do total de estudos analisados, a identificação de métodos de avaliação da qualidade dos dados do Sinasc possibilitou apontar alguns aspectos importantes que podem facilitar a replicação de estudos. O primeiro que deve ser considerado é que a avaliação da qualidade das informações contidas no Sinasc precisa tanto de avaliações de cobertura quanto de avaliações de completitude e de confiabilidade.

A cobertura relaciona-se à capacidade de obtenção da totalidade dos eventos ocorridos em uma região delimitada em um determinado espaço de tempo. Avaliar o grau de cobertura de uma base de dados pode ser realizado através da comparação com outra que seja considerada confiável ${ }^{3,18}$. Existem indícios que apontam limitações para a avaliação da cobertura do Sinasc a partir dos dados do registro civil ou de estimativas calculadas pelo $\mathrm{IBGE}^{10}$. Assim, propõe-se a avaliação através da busca ativa que considere fontes notificantes com alto potencial de melhoria para o sistema como o PACS (Programa agentes comunitários de saúde)/PSF (Programa Saúde da Família) (agentes comunitários de saúde como excelente fonte de informação sobre os eventos vitais, principalmente nos municípios menores e mais afastados dos grandes centros) e as parteiras tradicionais ${ }^{11,14}$. O Ministério da Saúde preconiza uma cobertura de $90 \%$ para o uso dos dados no cálculo direto de indicadores ${ }^{11}$, sendo o estudo do Maranhão ${ }^{13}$ o único desta revisão que esteve distante desse valor (em municípios de Minas Gerais ${ }^{10}$ e de Pernambuco ${ }^{11}$ foram encontrados valores próximos). A boa cobertura do Sinasc tem sido apontada por vários estudos em diversas áreas do país ${ }^{3,7}$, implicando a necessidade de aumentar a preocupação com a melhoria tanto da completitude das variáveis quanto da confiabilidade das informações.

A avaliação da completitude diz respeito ao preenchimento da DNV, em especial, à mensuração da frequência de informação "ignorada" ou em "branco". Variáveis ignoradas (não se conhece a informação) são produtos de uma série de deficiências como a ausência de informação nos prontuários e o desconhecimento de certas informações pelos acompanhantes da mulher, enquanto variáveis em branco (não preenchidas) são reflexo da falta de cuidado e de importância dada à DNV pelo profissional responsável por seu preenchimento, as variáveis que não se apli- cam deveriam ter uma codificação específica ou não constar no manual, pois parecem não ser necessárias².

O grau de preenchimento das variáveis pode ser categorizado considerando o sistema de escores proposto por Romero e Cunha ${ }^{7}$. A paridade, variável que mostrou maiores problemas de preenchimento, apresenta como características limitantes o fato de não existir uniformidade para seu registro (registra-se indistintamente o número de gestações anteriores ou o número de partos anteriores), além de que a DNV, nesse campo, emprega a denominação de nascidos mortos anteriores, o que pode trazer dúvidas para os serviços de saúde a respeito da informação que realmente deve ser registrada, apenas os nascimentos com mais de $1.000 \mathrm{~g}$ e/ou de 28 semanas e mais, ou todas as perdas fetais anteriores incluindo aqui os abortos espontâneos ${ }^{12}$.

A confiabilidade refere-se à concordância, consistência ou plausibilidade dos resultados considerando o pareamento das informações contidas na DNV com as informações de outros sistemas de informação, de pesquisas ou da utilização de uma Declaração de Nascido Vivo Controle $^{7,13}$. O grau de concordância pode ser obtido por meio do índice kappa ${ }^{19}$, no caso de variáveis qualitativas, e pelo coeficiente de correlação intraclass $^{20}$, em se tratando de variáveis quantitativas. As variáveis que apresentaram menores graus de concordância foram instrução materna, paridade e número de consultas de pré-natal. As variáveis instrução materna e número de consultas de pré-natal caracterizam-se por serem informações sobre as quais existe ausência de registro nos prontuários hospitalares, dependendo em muitas ocasiões de entrevista à puérpera para seu preenchimento ${ }^{3,13}$.

Por fim, a proposição de novas formas de avaliar o Sinasc, como estratégia privilegiada para a melhoria do sistema, deve considerar a importância de técnicas qualitativas que possibilitem analisar a importância atribuída ao sistema, assim como as dificuldades encontradas para o preenchimento da DNV e a análise do fluxo de informação. Considerando as fontes de dados para fins comparativos, a utilização de dados de pesquisas com coleta primária, a busca ativa $\mathrm{e}$ dados controle devem ser priorizados em comparação aos dados de fonte secundária. A importância de linkage (pareamento) entre bancos de dados enfatiza-se para o ganho na quantidade de informações. 


\section{Referências}

1. Theme Filha MM, Gama SGN, Cunha CB, Leal MC. Confiabilidade do Sistema de Informações sobre Nascidos Vivos Hospitalares no Município do Rio de Janeiro, 1999-2001. Cad Saude Publica 2004; 20(Supl. 1):S83-91.

2. Costa JMBS, Frias PG. Avaliação da completitude das variáveis da Declaração de Nascido Vivo de residentes em Pernambuco, Brasil, 1996 a 2005. Cad Saude Publica 2009; 25(3):613-624.

3. Mello Jorge MHP, Laurenti R, Gotlieb SLD. Análise da qualidade das estatísticas vitais brasileiras: a experiência de implantação do SIM e do Sinasc. Cien Saude Colet 2007; 12(3):643-654.

4. Brasil. Ministério da Saúde (MS). Manual de instruções para o preenchimento da declaração de nascido vivo. $3^{\text {a }}$ edição. Brasília: MS, Fundação Nacional de Saúde; 2001. [página na Internet]. [acessado 2012 set 3]. Disponível em: http://portal.saude.gov.br/ portal/arquivos/pdf/declaracao_nasc_vivo.pdf

5. Brasil. Ministério da Saúde (MS). Fundação Nacional de Saúde. Manual de procedimentos do Sistema de Informações sobre Nascidos Vivos. Brasília: MS; 2001.

6. Drumond EF, Machado CJ, Vasconcelos MR, França E. Utilização de dados secundários do SIM, Sinasc e SIH na produção científica brasileira de 1990 a 2006. $R$ bras Est Pop 2009; 26(1):7-19.

7. Romero DE, Cunha CB. Avaliação da qualidade das variáveis epidemiológicas e demográficas do Sistema de Informações sobre Nascidos Vivos, 2002. Cad Saude Publica 2007; 23(3):701-714.

8. Silva CF, Leite AJM, Almeida Nádia Maria Girão Saraiva de. Linkage entre bancos de dados de nascidos vivos e óbitos infantis em município do Nordeste do Brasil: qualidade dos sistemas de informação. Cad Saude Publica 2009; 25(7):1552-1558.

9. Guerra FAR, Llerena Jr JC, Gama SGN, Cunha CB, Theme Filha MM. Confiabilidade das informações das declarações de nascido vivo com registro de defeitos congênitos no Município do Rio de Janeiro, Brasil, 2004. Cad Saude Publica 2008; 24(2):438446.

10. Drumond EF, Machado CJ, França E. Subnotificação de nascidos vivos: procedimentos de mensuração a partir do Sistema de Informação Hospitalar. Rev Saude Publica 2008; 42(1):55-63.

11. Frias PG, Pereira PMH, Vidal AS, Lira PIC. Avaliação da cobertura do Sistema de Informações Sobre Nascidos Vivos e a contribuição de fontes potenciais de notificação do nascimento em dois Municípios de Pernambuco, Brasil. Epidemiol Serv Saúde 2007; 16(2):93-101.
12. Almeida MF, Alencar GP, Franca Júnior I, Novaes HMD, Siqueira AAF, Schoeps D, Campbell O, Rodrigues LC. Validade das informações das declarações de nascidos vivos com base em estudo de caso-controle. Cad Saude Publica 2006; 22(3):643-652.

13. Silva AAM, Ribeiro VS, Borba Júnior AF, Coimbra LC, Silva RA. Avaliação da qualidade dos dados do Sistema de Informações sobre Nascidos Vivos em 1997-1998. Rev Saude Publica 2001; 35(6):508-514.

14. Mello Jorge MHP, Gotlieb SLD. O Sistema de Informação de Atenção Básica como Fonte de Dados para os Sistemas de Informações sobre Mortalidade e sobre Nascidos Vivos. Inf Epidemiol SUS 2001; 10(1):7-18.

15. Misihima FC, Scochi CGS, Ferro MAR, Costa IAR. Declaração de nascido vivo: análise do seu preenchimento no Município de Ribeirão Preto, São Paulo, Brasil. Cad Saude Publica 1999; 15(2):387-395.

16. Mello Jorge MHP, Gotlieb SLD, Andrade SM. Análise dos registros de nascimentos vivos em localidade urbana no Sul do Brasil. Rev Saude Publica 1997; 31(1):78-89.

17. Mello Jorge MHP, Gotlieb SLD, Soboll MLMS, Almeida MF, Latorre MRDO. Avaliação do Sistema de Informação sobre Nascidos Vivos e o uso de seus dados em epidemiologia e estatísticas de saúde. Rev Saude Publica 1993; 27(6 Supl):1-46.

18. Souza LM. Avaliação do Sistema de Informação sobre Nascidos Vivos (Sinasc) - Minas Gerais - 2000. XIV Encontro Nacional de Estudos Populacionais; 2004 Set 20-24; Caxambu (MG): ABEP; 2004.

19. Dunn G. Design and analysis of reliability studies. New York: John Wiley \& Sons; 1989.

20. Szklo R, Nieto FJ. Epidemiology Beyond the Basis. Aspen: Aspen Publications; 2000.

Artigo apresentado em 22/06/2011

Aprovado em 15/11/2011

Versão final apresentada em 02/01/2012 
